Apidologie, 1978, 9 (2), 101-110.

\title{
ACTION DE DEUX INSECTICIDES SUR MELITTOBIA ACASTA WALK. ET SON HOTE : \\ MEGACHILE PACIFICA PZ. (HYMENOPTERA EULOPHIDAE, MEGACHILIDAE). MÉTHODE DE PRÉVENTION DU PARASITISME
}

\author{
Die Wirkung von zwei Insektiziden auf Melittobia acasta Walk. \\ und ihren Wirt: Megachile pacifica Panz. \\ (Hymenoptera Eulophidae, Megachilidae. \\ Methoden zur Verhinderung der Parasitierung
}

\author{
J.-N. TASEI \\ avec la collaboration technique de S. CARRE \\ Laboratoire de Zoologie, \\ S.A.P.F., I.N.R.A., 86600 Lusignan
}

\section{SUMMARY}

EFFECTS OF 2 INSECTICIDES ON MELITTOBIA ACASTA WALK AND ITS HOST : $M E G A C H I L E$ PACIFICA PZ (HYMENOPTERA, EULOPHIDAE, MEGACHILIDAE). A METHOD FOR PARASITE CONTROL.

Tests in controlled conditions were used to compare the efficiency of endosulfan (Thiodan) and carbaryl (Sevin) for destroying the parasite : Melittobia acasta during the incubation of the alfalta leaf cutter bees. All the parasites are killed 8 hours after emergence till the eighteenth day of incubation at $30^{\circ}$ thanks to the soaking of cells in insecticide solutions. This efficiency is reached above the concentration levels : $4 \%$ or endosulfan and $1 \mathrm{~g} / \mathrm{l}$ for carbaryl. No bees are killed before emergence but adults, particularly males can die after emergence. A $1 \mathrm{~g} / \mathrm{l}$ carbaryl solution is less hazardous to adult bees than $4 \%$ endosulfan and is recommended for treatment of commercial cells.

\section{RÉSUMÉ}

Des tests en condition contrôlées ont permis de comparer l'efficacité de l'endosulfan (Thiodan) et du carbaryl (Sevin) pour détruire le parasite : Melinobia acasta pendant la phase de réactivation des prénymphes de mégachiles. Le trempage des cellules au sortir de l'hibernation dans les solutions insecticides élimine en 8 heures pratiquement tous les parasites jusqu'au $18^{\mathrm{c}}$ jour d'incubation à $30^{\circ} \mathrm{C}$. Cette efficacité est atteinte au-dessus des concentrations : $4 \%$ pour l'endosulfan et $1 \mathrm{~g} / 1$ pour le carbaryl. Aucun dommage n'est causé aux abeilles à l'intérieur des cocons mais des pertes sont observées chez les adultes émergeant, surtout les mâles. Le carbaryl à $1 \mathrm{~g} / \mathrm{l}$ est moins dangereux pour les adultes que l'endosulfan à $4 \%$; il est recommandé pour traiter les lots commerciaux de cellules. 


\section{INTRODUCTION}

Dans les publications précédentes sur l'élevage en champ des mégachiles de la luzerne : Megachile pacifica Pz. (TASEI 1975, 1977 a, 1977 B) on a attiré l'attention sur les dangers que font courir aux larves certains insectes prédateurs ou parasites, en raison de leur voracité ou de leur abondance. Parmi les parasites le petit hyménoptère Melittobia acasta menace les populations de mégachiles aussi bien pendant les trois semaines de la phase de réactivation des prénymphes qui se déroule dans un incubateur à $30^{\circ}$ constants, que pendant la période de trois mois correspondant au vol estival des deux générations annuelles.

L'étude de la biologie du parasite par Balfour Brown (1922) et quelques observations personnelles fournissent les explications de la gravité des attaques qui peuvent aller jusqu'à la perte totale de la population. Pendant l'hibernation les Melittobia sont au repos, soit à l'état de larves, soit à l'état de nymphes plus ou moins pigmentées. Ce sont ces dernières qui donnent les premiers adultes dès le cinquième jour d'incubation à $30^{\circ} \mathrm{C}$, puis à partir du dixième jour émergent les adultes issus des larves hibernantes. Le jour même de leur émergence les Melittobia femelles sont capables de percer des cellules saines et de pondre plusieurs dizaines d'œufs sur chaque larve ou nymphe d'abeille. La rapidité du développement à la température de l'incubateur permet à la deuxième génération de sortir des cocons aux environs du vingtième jour c'est-à-dire en même temps que les premières abeilles. Ces individus feront alors souche dans les nichoirs à l'intérieur des cellules fraîchement construites. Une troisième génération aura tout loisir de parasiter les cocons durant l'été, d'autant plus aisément que les pièces de bois constituant le nichoir sont mal ajustées et laissent des interstices, qui sont pour les femelles des points de passage d'un nid à l'autre.

On sait qu'il est impossible d'enrayer les invasions de Melittobia par le seul usage de pièges à rayonnement ultra-violet (BoHART, 1972). C'est pour cette raison que certains auteurs ont eu l'idée d'éliminer les parasites en immergeant les cellules de mégachiles dans une solution de carbaryl. Après séchage, les cellules sont recouvertes d'un film insecticide qui les protège contre le parasitisme et n'entraine pas de mortalité excessive chez les abeilles (BrindLey, 1976). Dès le printemps 1976, j'ai essayé avec succès une technique analogue qui consistait à pulvériser sur les cellules sortant d'hibernation une solution à $4 \%$ o d'endosulfan (TASEI, 1977 b). Le présent travail est destiné à comparer l'action du carbaryl et de l'endosulfan dans les mêmes conditions, aussi bien sur les adultes de Melittobia que sur les larves et les adultes de mégachiles. Ces bases expérimentales permettront d'opter pour un système de traitement des lots commerciaux de cellules.

\section{MATÉRIEL ET MÉTHODES}

Deux tests doivent être mis en œuvre afin de voir l'action du carbaryl et de l'endosulfan sur le parasite et son hôte. 


\section{1. - Action des insecticides sur Melittobia.}

Les produits utilisés sont l'endosulfan sous forme de Thiodan E.C. à $365 \mathrm{~g} / 1$ d'endosulfan et le carbaryl sous forme de Sevin L 85 à $85 \%$ de carbaryl. Après quelques expériences préliminaires que je ne relate pas ici on adopte le protocole suivant: $\mathrm{C}_{4}$.

Quatre concentrations de produit commercial sont choisies pour chacun des insecticides : $\mathrm{C}_{1}, \mathrm{C}_{2}, \mathrm{C}_{3}$,

Pour l'endosulfan $=\mathrm{C}_{1}=4 \%$ o $\quad \mathrm{C}_{2}=2 \% ; \quad \mathrm{C}_{3}=1 \% ; \quad \mathrm{C}_{4}=0,5 \%$ o.

Pour le carbaryl $=\mathrm{C}_{1}=2 \mathrm{~g} / \mathrm{l} ; \quad \mathrm{C}_{2}=1,5 \mathrm{~g} / \mathrm{l} ; \quad \mathrm{C}_{3}=1 \mathrm{~g} / \mathrm{l} ; \quad \mathrm{C}_{4}=0,5 \mathrm{~g} / \mathrm{l}$.

On teste l'efficacité des deux produits aux quatre concentrations, 8 jours, 13 jours et 18 jours après le traitement des cocons.

Les Melittobia proviennent de la deuxième génération d'un élevage de masse pratiqué sur des larves de guêpes Eumenidae.

Des cellules de mégachiles sont trempées dans les huit solutions insecticides citées plus haut. Elles sont agitées doucement pendant $30 \mathrm{~s}$ afin que toute la surface soit bien humectée. Les cellules témoins sont trempées dans l'eau. Après un séchage de 6 à 7 heures à $18-20^{\circ} \mathrm{C}$ on place les cellules traitées en étuve à $30^{\circ} \mathrm{C}$, ayant une humidité relative de $60-70 \%$ et contenant une lampe à rayon U.V. de $6 \mathrm{~W}$. Trois séries de huit traitements insecticides sont nécessaires. La première a lieu 18 jours avant le test, la seconde 13 jours avant, la dernière 8 jours avant.

Le jour du test on introduit dans des boites en polystyrène cristal (de $9 \times 6 \times 2 \mathrm{~cm}$ ) une demidouzaine de cocons de chaque catégorie puis on laisse tomber 10 à 30 Melittobia âgées de moins de 2 jours dans chaque boite dont on scelle le couvercle avec une bande de papier adhésif. Toutes les boites sont alors portées dans l'étuve à $29-30^{\circ} \mathrm{C}$ et on dénombre les cadavres $\mathrm{I}$ heure, 4 heures, 6 heures, 8 heures après l'introduction. Chaque traitement est répété quatre fois ce qui nécessite l'emploi de 100 boites et de 1755 individus de Melittobia. On peut noter que dans cette population se trouvaient seulement 37 mâles.

\section{2. - Action des insecticides sur les mégachiles.}

Un total de 3200 cocons issus de l'élevage en champ de 1976 sont sortis d'hibernation et trempés $30 \mathrm{~s}$ par lots de 60 à 70 , soit dans l'eau pour le témoin, soit dans des solutions d'endosulfan ou de carbaryl aux concentrations $\mathrm{C}_{1}, \mathrm{C}_{2}, \mathrm{C}_{3}, \mathrm{C}_{4}$ déjà indiquées. Chaque traitement est répété quatre fois. Après un séchage de 6 à 7 heures à $18-20^{\circ} \mathrm{C}$ tous les lots, soit 48 boîtes en polystyrène identiques à celles que l'on utilise dans le paragraphe 1 sont mises en l'étuve à $21-30^{\circ} \mathrm{C}$. Au bout de 20 jours les adultes commencent à émerger. Chaque jour les mâles et les femelles sont retirés des boites en polystyrène et placés dans des boites à couvercle grillagé laissées à température de laboratoire à l'abri de la lumière. Chaque jour on effectue un comptage des individus morts. Les pourcentages de mortalité sont calculés dès que $90 \%$ des mâles et $75 \%$ des femelles sont sortis, soit au $7^{\mathfrak{e}}$ jour de la période d'émergence de chaque lot. Cette méthode à l'intérêt de ne prendre en compte que les individus à sortie précoce qui sont en contact avec des résidus insecticides âgés de 20 à 27 jours et atténue les conséquences d'un séjour prolongé sans nourriture.

Après la sortie de tous les insectes, abeilles et parasites (Coelioxys, Anthrax, Leucospis, Monodontomerus, Melittobia) on compte dans chaque lot les cocons renfermant une larve ou une nymphe morte, afin de voir si l'effet des trempages cause du tort aux stades immatures.

\section{RÉSULTATS}

\section{1. - Action des insecticides sur les Melittobia.}

Sur la figure 1 (A - B - C) on constate que c'est au bout de 8 heures que l'on obtient l'élimination complète des Melittobia dans les meilleurs cas. Il faut signaler que les lots témoins sont restés vivants à $100 \%$ dans des conditions identiques. 


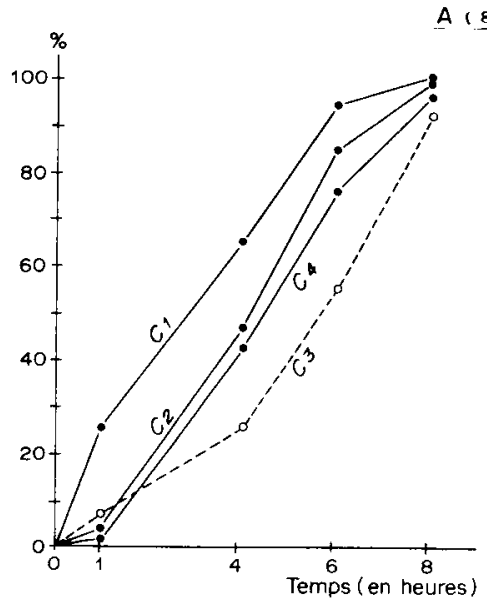

A ( 8 IOURS)
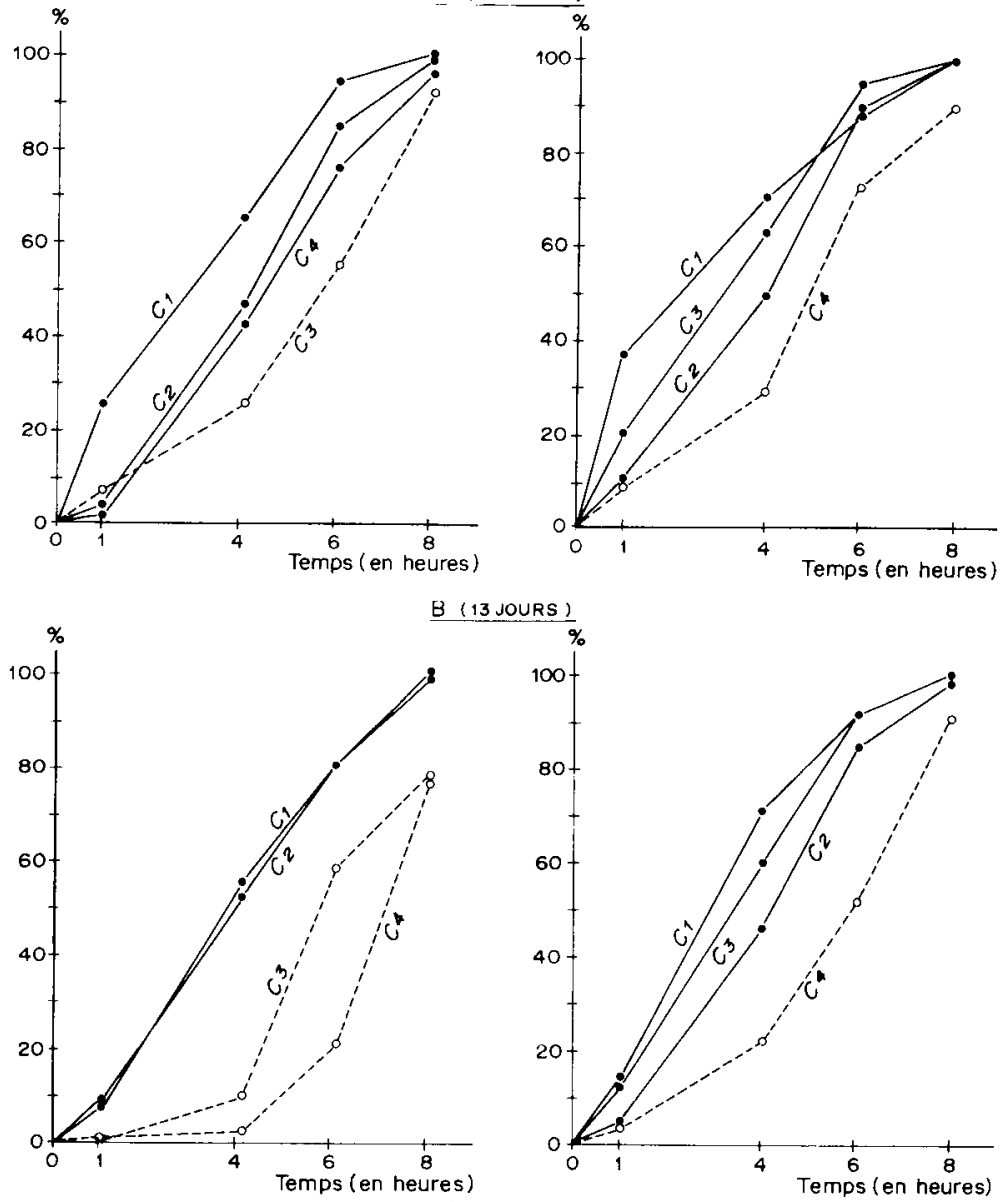

(13 JOURS)

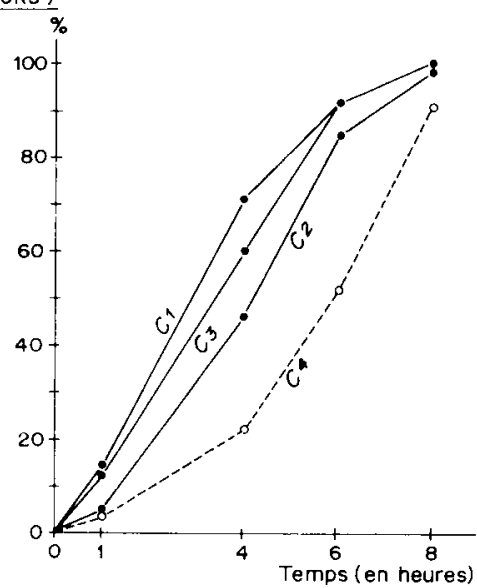

C (18 JOURS)
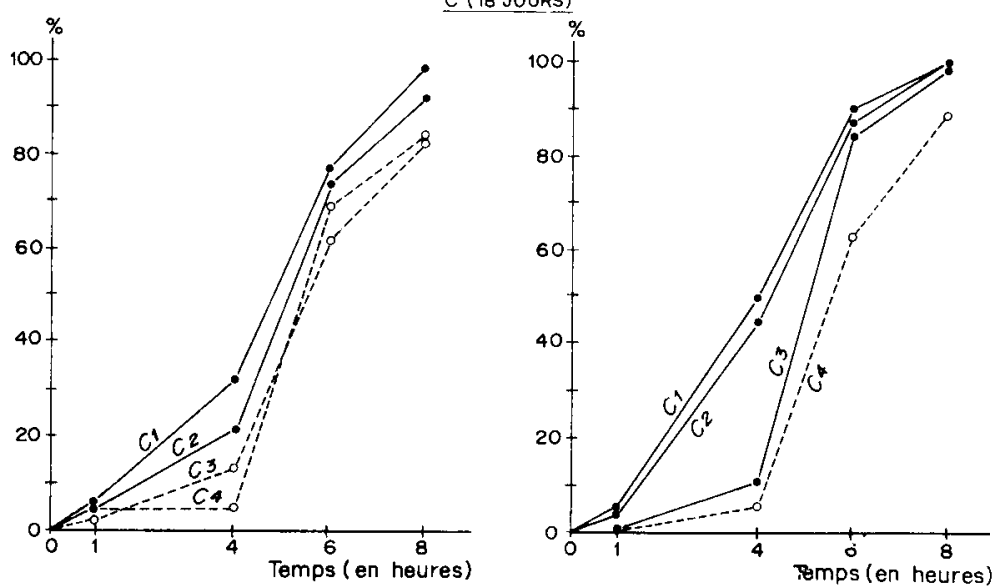
FIG. 1. - Action de 2 insecticides sur Melitiobia acasta.

Verticalement : pourcentage de mortalité.

Horizontalement : durée du test er heures.

$\mathrm{C}_{1}=$ concentration $4 \%$ pour l'Endosulfan, $2 \mathrm{~g} / \mathrm{l}$ pour le Carbaryl;

$\mathrm{C}_{2}=$ concentration $2 \%$ pour l'Endosulfan, $1,5 \mathrm{~g} / 1$ pour le Carbaryl;

$\mathrm{C}_{3}=$ concentration $1 \%$ pour l'Endosulfan, 1 g/l pour le Carbaryl;

$\mathrm{C}_{4}=$ concentration $0,5 \%$ pour l'Endosulfan, $0,5 \mathrm{~g} / \mathrm{l}$ pour le Carbaryl.

$\bullet-\bullet$ : courbes confondues du point de vue statistique

: courbes différant des précédentes par au moins 2 points. (Seuil de probabilité =0,1.)

$\mathrm{A}=$ âge des résidus : 8 jours.

$\mathrm{B}=$ âge des résidus : 13 jours.

$\mathrm{C}=$ âge des résidus : 18 jours.

FIG. 1. - Wirkung zweier Insektizide auf Melittobia acasta.

Senkrechte : Sterblichkeit in Prozent.

Waagerechte : Testdauer in Stunden.

$\mathrm{C}_{1}=$ Konzentration von $4 \%$ für Endosulfan, von $2 \mathrm{~g} / \mathrm{l}$ für Carbaryl;

$\mathrm{C}_{2}=$ Konzentration von $2 \%$ für Endosulfan, von $1,5 \mathrm{~g} / 1$ für Carbaryl;

$\mathrm{C}_{3}=$ Konzentration von $1 \%$ für Endosulfan, von $1 \mathrm{~g} / 1$ für Carbaryl;

$\mathrm{C}_{4}=$ Konzentration von $0,5 \%$ oür Endosulfan, von $0,5 \mathrm{~g} / 1$ für Carbaryl.

$-\bullet$ : vermischte Kurven aus statistischer Sicht

: Kurven, die sich von den vorhergehenden um mindestens zwei Punkte unterscheiden (Irrtumswahrscheinlichkeit $=0,1$ ).

$\mathrm{A}=$ Alter der Beläge : 8 Tage.

$\mathrm{B}=$ Alter der Beläge : 13 Tage.

$\mathrm{C}=$ Alter der Beläge : 18 Tage. 
L'endosulfan comme le carbaryl sont de très bons insecticides dont l'action persiste après 18 jours d'étuve à $30^{\circ} \mathrm{C}$. Cependant ils se comportent de façon légèrement différente selon l'âge des résidus. On voit sur la figure 1 que les concentrations $C_{3}$ et $C_{4}$ de l'endosulfan sont significativement moins actives que $C_{1}$ dans les tests de rémanence de 13 et 18 jours $(B-C)$ et que la concentration $C_{4}$ du carbaryl est significativement moins active que les trois autres, quel que soit l'âge des résidus.

Si l'on se refère au tableau 1 on constate :

a) Après 8 heures de contact les résidus âgés de 18 jours éliminent plus de $95 \%$ des individus à condition d'employer les concentrations $\mathrm{C}_{1}, \mathrm{C}_{2}$ ou $\mathrm{C}_{3}$ pour le carbaryl et $\mathrm{C}_{1}$ ou $\mathrm{C}_{2}$ pour l'endosulfan.

b) Si l'on s'en tient au délai d'action de 6 heures l'élimination à $95 \%$ n'a lieu que pour les résidus âgés de 8 jours seulement et à condition d'employer les concentrations $\mathrm{C}_{1}, \mathrm{C}_{2}$ ou $\mathrm{C}_{3}$ pour le carbaryl et la concentration $\mathrm{C}_{1}$ pour l'endosulfan.

c) Lorsque les résidus sont âgés de 13 et 18 jouĩs les doses $C_{1}, C_{2}$ ou $C_{3}$ du carbaryl éliminent après 6 heures d'action 85 à $95 \%$ des Melittobia alors que l'endosulfan est nettement moins efficace.

\section{2. - Action des insecticides sur les mégachiles.}

A l'issue du test de survie des adultes après émergence on a démombré 1250 femelles et 1470 mâles. Seuls les individus émergeant pendant les 7 premiers jours, soit 950 femelles et 1325 mâles ont servi aux calculs des taux de mortalité. Selon la figure 2 les principaux résultats du test sont les suivants :

TABL. 1. - Concentration d'Endosulfan et de Carbaryl éliminant plus de $95 \%$ des Melittobia. TĄB. 1. - Die Konzentration von Endosulfan und Carbaryl, die mehr als $95 \%$ der Melittobia beseitigt.

\begin{tabular}{|c|c|c|c|c|}
\hline \multirow[b]{2}{*}{$\begin{array}{l}\text { Age des résidus } \\
\text { Alter der Beläge }\end{array}$} & \multicolumn{2}{|c|}{$\begin{array}{l}6 \text { heures } \\
6 \text { Stunden }\end{array}$} & \multicolumn{2}{|c|}{$\begin{array}{l}8 \text { heures } \\
8 \text { Stunden }\end{array}$} \\
\hline & Endosulfan & Carbaryl & Endosulfan & Carbaryl \\
\hline $\begin{array}{l}8 \text { jours } \\
8 \text { Tage }\end{array}$ & $\mathrm{C}_{1}$ & $\begin{array}{l}C_{1} \\
C_{2} \\
C_{3}\end{array}$ & $\begin{array}{l}C_{1} \\
C_{2} \\
C_{3} \\
C_{4}\end{array}$ & $\begin{array}{l}C_{1} \\
C_{2} \\
C_{3}\end{array}$ \\
\hline $\begin{array}{l}13 \text { jours } \\
13 \text { Tage }\end{array}$ & & & $\begin{array}{l}\mathrm{C}_{1} \\
\mathrm{C}_{2}\end{array}$ & $\begin{array}{l}\mathrm{C}_{1} \\
\mathrm{C}_{2} \\
\mathrm{C}_{3}\end{array}$ \\
\hline $\begin{array}{l}18 \text { jours } \\
18 \text { Tage }\end{array}$ & & & $C_{1}$ & $\begin{array}{l}\mathrm{C}_{1} \\
\mathrm{C}_{2} \\
\mathrm{C}_{3}\end{array}$ \\
\hline
\end{tabular}




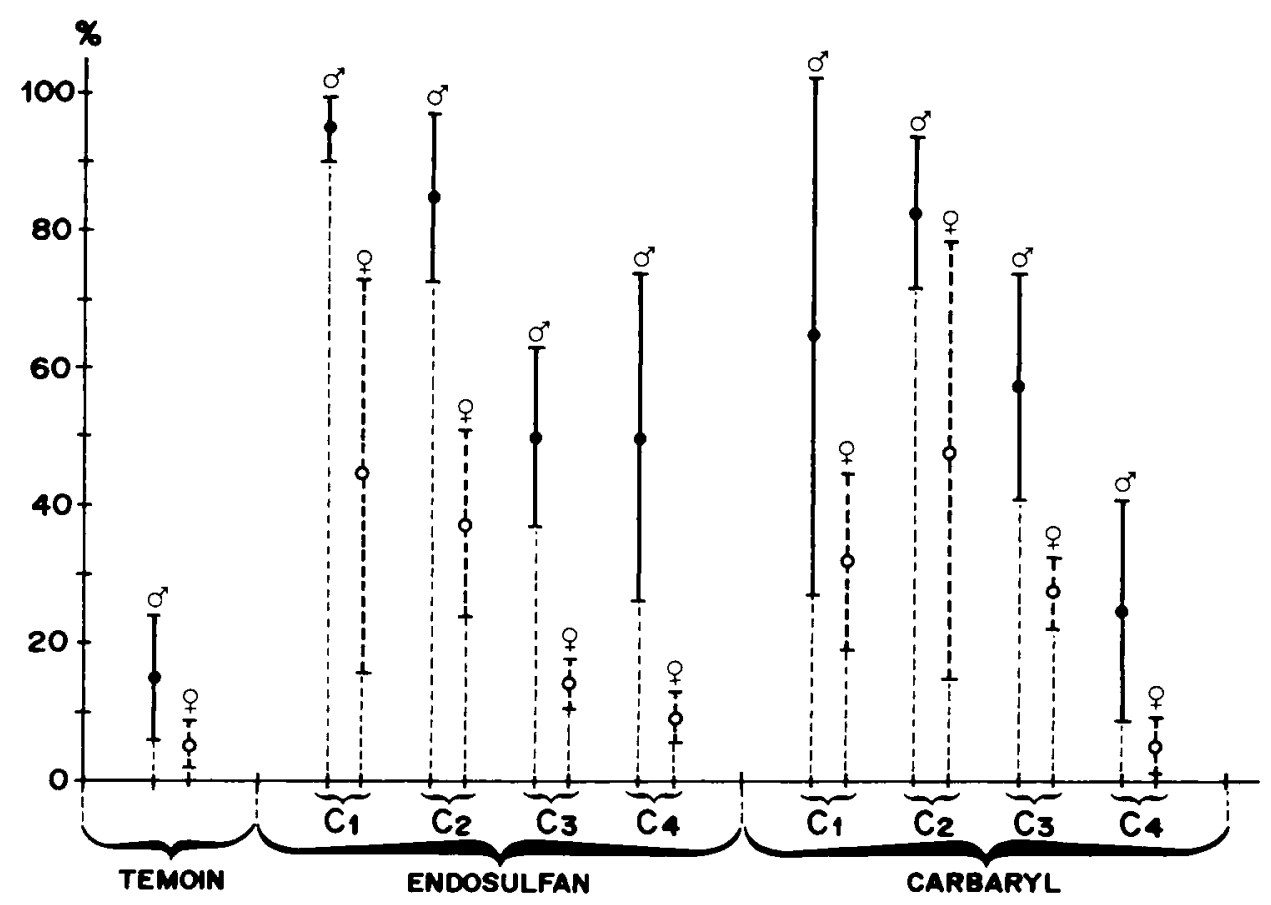

FIG. 2. - Effet du trempage des cocons dans des solutions insecticides sur la mortalité des mégachiles à l'émergence $3 \grave{a} 4$ semaines après le traitement.

Verticalement : pourcentage de mortalité.

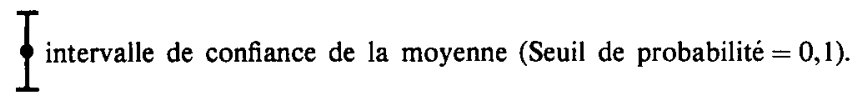

FIG. 2. - Auswirkung des Eintauchens der Kokons in die insektiziden Lösungen auf die Sterblichkeit der Blattschneiderbienen beim Schlüpfen, 3 bis 4 Wochen nach der Behandlung

Senkrechte : Sterblichkeit in Prozent. Vertrauensintervall des Mittelwertes (Irrtumswahrscheinlichkeit $=0,1)$.

a) La mortalité du témoin est statistiquement identique chez les mâles et les femelles.

b) La sensibilité des mâles aux résidus insecticides est plus grande que celle des femelles. Ce phénomène est net dans le cas de l'endosulfan pour les quatre concentrations, il est moins perceptible pour le carbaryl.

c) Les traitements à l'endosulfan et au carbaryl sont inoffensifs pour les femelles à la seule concentration $\mathrm{C}_{4}$. La mortalité des mâles est identique à celle du témoin dans le cas du carbaryl mais elle lui est légèrement supérieure dans le cas de l'endosulfan. Les concentrations $C_{3}$ et $C_{4}$ de l'endosulfan ont une action assez proche l'une de l'autre.

d) Les concentrations $\mathrm{C}_{1}, \mathrm{C}_{2}$ et $\mathrm{C}_{3}$ du carbaryl ont des toxicités statistiquement identiques vis-à-vis des mâles et des femelles et supérieures à $C_{4}$. Les concentrations $C_{1}$ et $C_{2}$ de l'endosulfan sont plus toxiques que la concentration $C_{3}$ et a fortiori $C_{4}$. 
TABL. 2. - Mortalité des larves et des nymphes dans les cocons traités par trempage dans les solutions insecticides Résultats exprimés en pourcentage (Seuil de probabilité $=0,1$ ).

Тав. 2. - Sterblichkeit der Larven und Puppen in den Kokons, die durch Eintauchen in Insektizidlösungen behandelt wurden.

Die Ergebnisse werden in Prozent dargestellt (Irrtumswahrscheinllehkeit $=0,1$ ).

\begin{tabular}{|c|c|c|c|}
\hline $\begin{array}{l}\text { Concentration } \\
\text { Konzentration }\end{array}$ & Endosulfan & Carbaryl & $\begin{array}{l}\text { Témoin } \\
\text { Kontrolle }\end{array}$ \\
\hline 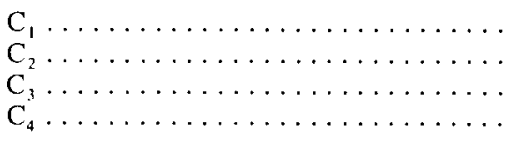 & $\begin{array}{r}12,3 \pm 4 \\
6,5 \pm 3 \\
5,6 \pm 1.9 \\
10.6 \pm 4.7\end{array}$ & $\begin{array}{l}4,2 \pm 1,2 \\
4,6 \pm 2,6 \\
6.4 \pm 3.2 \\
8,8 \pm 1,9\end{array}$ & $\begin{array}{l}6,4 \pm 3,7 \\
3,9 \pm 2,8 \\
3,6 \pm 6,0 \\
7,3 \pm 2,9\end{array}$ \\
\hline $\begin{array}{l}\text { Moyenne } \ldots \ldots \ldots \ldots \ldots \\
\text { Mittelwert }\end{array}$ & $8,8 \pm 2,1$ & $6,0 \pm 1.4$ & $5.4 \pm 1,9$ \\
\hline
\end{tabular}

e) Le pourcentage de cocons où l'on trouve une larve ou une nymphe morte varie de 3,6 à 12,3 suivant le lot. Aucun test statistique $\left(\mathrm{x}^{2}\right.$, test de $\left.t\right)$ ne permet de détecter une différence significative de mortalité de stades immatures, même aux plus fortes concentrations, entre les lots traités et les lots témoins (Tableau 2).

\section{DISCUSSION - CONCLUSION}

La protection des cocons de mégachiles durant l'incubation contre une attaque de Melittobia nécessite l'éradiction de ces insectes entre le $5^{\mathrm{e}}$ jour et le $18^{\mathrm{e}}$ jour d'incubation à $29-30^{\circ} \mathrm{C}$, la mort des parasites devant arriver dans les huit heures suivant leur sortie. D'après les résultats (Fig. $1 \mathrm{C}$ et tableau 1) l'endosulfan à la concentration $C_{1}$ et le carbaryl aux concentrations $C_{1}, C_{2}$ ou $C_{3}$ sont capables d'éliminer ṕlus de $95 \%$ des Melittobia après 8 heures de contact et ceci le $18^{\mathrm{e}}$ jour d'incubation. Cependant les risques plus élevés pour les abeilles, particulièrement les mâles, lors du contact avec les cocons traités aux concentrations $C_{1}$ de l'endosulfan et $\mathrm{C}_{1}$ et $\mathrm{C}_{2}$ du carbaryl font choisir la concentration $\mathrm{C}_{3}(1 \mathrm{~g} / 1)$ du carbaryl.

Même à la concentration de $1 \mathrm{~g} / \mathrm{l}$, les résidus de carbaryl provoquent une mortalité supérieure à celle des abeilles témoins. Dans les conditions naturelles les pertes ne pourront qu'être nettement inférieures aux $28 \%( \pm 4)$ de femelles et $57 \%( \pm 17)$ de mâles (Fig. 2), pourcentages qui sont obtenus dans des conditions sévères, soit 1 à 7 jours de claustration sans nourriture après émergence du cocon.

Il est donc recommandé de traiter préventivement les cellules de mégachiles le jour de leur sortie du réfrigérateur. On les trempe dans une solution de carbaryl à $1 \mathrm{~g} / \mathrm{l}$ 
pendant $30 \mathrm{~s}$ en remuant doucement afin que toute la surface soit bien mouillée. Après égouttage et séchage à température de laboratoire pendant 6 à 12 heures on réactive les prénymphes en incubateur à $30^{\circ} \mathrm{C}$. Cette technique a donné satisfaction en 1977 lors de l'installation au champ de 40000 cellules.

Ce traitement ne semble pas atteindre les genres suivants: Monodontomerus, Coelioxys, Miltogramma, Anthrax. Une étude ultérieure devrait permettre de trouver une solution analogue à celle qui a été trouvée pour les Melittobia.

Reçu pour publication en octobre 1977. Eingegangen im Oktober 1977.

\section{REMERCIEMENTS}

Les firmes : La Littorale et Procida ont eu l'amabilité de faire parvenir au laboratoire des échantillons de Thiodan et de Sevin qui ont permis de réaliser ce travail.

\section{ZUSAMMENFASSUNG}

Die vorausgegangenen Veröffentlichungen, die Freilandzucht der Luzerne-Blattschneiderbiene betreffend (TASEI 1975, 1977 a, 1977 b), betonen die Gefahren, die gewisse Räuber und Parasiten den Populationen dieser Bienen zufügen können, besonders Melittobia acasta. Diese kleine Hymenoptere schlüpft zwischen dem 5. und 10. Tag der Ruhezeit bei $29-30^{\circ}$, wird nur schwach von den UV-Lichtfallen angelockt und man versucht, auf Insektizidbehandlungen zurückzugreifen, um die Melittobia zu beseltgen. Gewisse Erfahrungen wurden im Labor mit Carbaryl gewonnen (BRindLey 1976), andere unter Freilandbedingungen mit Endosulfan (TASEI 1977 b). Durch einen Vergleich dieser beiden Insektizide unter kontrollierten Bedingungen soll die vorliegende Arbeit die notwendigen Kenntnisse liefern für eine rationelle Behandlung der im Handel erhältlichen Kokons.

\section{MATERIAL UND METHODEN}

Die verwendeten handelsüblichen Produkte sind Thiodan ( $365 \mathrm{~g}$ Endosulfan pro Liter) und Sevin ( $85 \%$ Carbaryl) und vier Konzentrationen des Handelspräparates wurden zur Behandlung der Kokona verwendet : $\mathrm{C}_{1}, \mathrm{C}_{2}, \mathrm{C}_{3}, \mathrm{C}_{4}$. Bei Endosulfan $\mathrm{C}_{1}=4 \%$ o, $\mathrm{C}_{2}=2 \%$ o, $\mathrm{C}_{3}=1 \%, \mathrm{C}_{4}=0,5 \%$. Bei Carbaryl $\mathrm{C}_{1}=2 \mathrm{~g} / \mathrm{l}, \mathrm{C}_{2}=1,5 \mathrm{~g} / \mathrm{l}, \mathrm{C}_{3}=1 \mathrm{~g} / \mathrm{l}, \mathrm{C}_{4}=0,5 \mathrm{~g} / \mathrm{l}$. Man prüft die Wirksamkeit der beiden Produkte 8,13 und 18 Tage nach der Behandlung der Kokons. Die Kokons der Blattschneiderbienen werden in die acht Insektizidlösungen eingetaucht, dann $30 \mathrm{sec}$ bewegt, dann abtropfen gelassen und 6-7 Stunden bei 18-20 getrocknet. Die Kokons werden in einer Trockenkammer bei $30^{\circ}$ untergebracht, 8,13 oder 18 Tage vor dem Testtag. Am Testtag gibt man 6 behandelte Kokons zusammen mit 10 bis 30 Melittobia in kleine Gefässe. Jeder Versuch wird viermal wiederholt. Das Auszählen der toten Tiere erfolgt 1, 4, 6 und 8 Stunden nach dem Einsetzen.

Ein zweiter Test dient dazu, die Wirkung der Insektizide auf die Bienen zu messen. In diesem Fall verwendet man 60 bis 70 behandelte Kokons, die eine Inkubationszeit von etwa 3 Wochen bei $29-30^{\circ}$ durchlaufen. Dann entmimmt man jeden Tag die geschlüpften Imagines und bringt sie in Kästen unter, die unter Lichtabschluss bei Labortemperatur verbleiben. Dic Sterblichkeitsrate wird errechnet, wenn $90 \%$ der Männchen und $75 \%$ der Weibchen geschlüpft sind, etwa am 7, Tag der Schlupfperiode jeder Kokonmenge. 


\section{ERGEBNISSE}

1. Endosulfan und Carbaryl sind bis zum 18. Tag in der Trockenkammer bei $30^{\circ}$ gegen Melittobia wirksam. Hinsichtlich der Konzentrationen verhalten sich die beiden Insektizide unterschiedlich (siehe Abb. 1 und Tab. 1).

2. Nach 8 Stunden Kontakt töten die 18 Tage alten Beläge über $95 \%$ der Individuen unter der Voraussetzung, dass man bei Carbaryl die Konzentrationen $C_{1}, C_{2}$ oder $C_{3}$ verwendet und bei Endosulfan die Konzentration $C_{1}$.

3. Nach 6 Stunden Kontakt gilt die Abtötung von $95 \%$ nur für die 8 Tage alten Beläge unter der Voraussetzung, dass man für Carbaryl die Konzentrationen $\mathrm{C}_{1}, \mathrm{C}_{2}$ oder $\mathrm{C}_{3}$ verwendet und fûr Endosulfan die Konzentration $\mathrm{C}_{1}$.

4. In Abb. 2 stellt man fest, dass die Sterblichkeit der Imagines der Blattschneiderbienen bei den Kontrollen für Männchen und Weibchen statistisch gleich ist.

5. Die Empfindlichkeit der Männchen gegenüber insektiziden Belägen ist grösser als die der Weibchen.

6. Nur bei der Konzentration $\mathrm{C}_{4}$ sind die Behandlungen unwirksam.

7. Tab. 2 zeigt, dass bei den Entwicklungsstadien (Larven oder Puppen) der Bienen kein Unterschied in der Sterblichkeit der Kontrollen und der behandelten Proben besteht, und zwar unabhängig von der Konzentration des Insektizids.

\section{SCHLUSSFOLGERUNGEN}

Die Schutzbehandlung der Kokons bewirkt die völlige Beseitigung der Melittobia zwischen dem 5. und 18. Tag der Inkubationszeit bei $29-30^{\circ}$, die Abtötung der Parasiten erfolgt innerhalb von 8 Stunden nach ihrem Schlupf. Andererseits schadet die Behandlung der Kokons gegen Ende der Winterruhe den Bienen nicht, wenn sie ab dem 20. Tag schlüpfen. Die Behandlung, die diese beiden Bedingungen zu erfüllen scheint, besteht in einem Eintauchen der Kokons in eine Carbaryllösung von $1 \mathrm{~g} / \mathrm{l}$ des Handelsproduktes Sevin L 85. Diese Technik schützt aber nur gegen die Angriffe der Melittobia, die anderen Parasiten scheinen durch die Behandlung mit Carbaryl nicht geschädigt zu werden.

\section{BIBLIOGRAPHIE}

Balbour Browne F, 1922. - On the life history of Melittobia acasta Walk. A chalcid parasite of bees and wasps. Parasitology 14, 349-370.

BoharT G.E., 1972. - Management of wild bees for the pollination of crops. Annu. Rev. Entomol. 17, 287-312.

BRINDLEY W.A., 1976. - Carbaryl control of chalcidoid parasites from alfalfa leaf cutting bees. J. Econ. Entomol. 69, 225-228.

TASEI J.N., 1975. - Le problème de l'adaptation de Megachile (eutricharaea) pacifica Panz. (Megachilidae) américain en France. Apidologie 6, 1-57.

TASEI J.N., 1977 (a). - Possibilité de multiplication du pollinisateur de la luzerne Megachile pacifica Panz. en France. Apidologie 8, 61-82.

TASEI J.N., 1977 (b). - Élevage d'un insecte pollinisateur de la luzerne dans le Midi de la France : Megachile pacifia Panz. (= Megachile rotundata F.) (Hym. Megachilidae). Cinquièmes journées Phvtiatr. Phytopharm. Circum-Méditerranéennes. 15-20 mai 1977. 